\title{
Guidelines for TESS Aperture Photometry via AstrolmageJ
}

\author{
JeREMy HA, ${ }^{1, *}$ AND PAT BOYCE ${ }^{2}$ \\ ${ }^{1}$ San Dieguito Academy High School, Encinitas, California, United States of America \\ ${ }^{2}$ Boyce Research Initiatives and Research Foundation, San Diego, California, United States of America \\ *Corresponding author: jeremyjha@gmail.com
}

Observing exoplanets with small telescopes can be a challenging but rewarding undertaking. With the advent of the Transiting Exoplanet Survey Satellite (TESS), a student observer can become qualified to participate in this exciting MIT-led NASA program to make significant contributions to exoplanet science. This paper presents the experiences and recommendations for a student to the complete observation data required for the Ground-Based Subgroup 1 TESS Follow-up Observing Program (TFOP). A training plan is provided along with various websites and instructional documents. Explanations on how apply the AstroImageJ (AIJ) software and procedures are described in A Practical Guide to Exoplanet Observing (Conti, 2018). Data is collected from Las Cumbres Observatory (LCO) and calibrated through the OSS Pipeline. This paper deals exclusively with LCO processes and also describes how to prepare the twelve files required for submission to the Exoplanet Follow-up Observing Program for TESS (ExoFOP - TESS). The AstroImageJ Guide for LCO - TESS Observations (Boyce et al., 2019) that incorporates the latest TFOP SG1 Observation Guidelines (Conti, 2019) is also referenced.

(ㅁ) $\circledast € 2020$ Astronomy Theory, Observations and Methods Journal

Keywords: $\quad$ MIT TESS - methods: differential photometry, data analysis - techniques: transit modeling - software: AstroImageJ

https://doi.org/10.32374/atom.2020.1.7

\section{INTRODUCTION}

This paper addresses the data reduction of TESS exoplanet observations from the Las Cumbres Observatory that are processed through the OSS Pipeline (Fitzgerald, 2018) for submission to the TESS ExoFOP. This process can be convoluted and arduous, which is where this paper's purpose lies. It supplements the already existing guides on data reduction with counseling on the clearest, most efficient ways to un- dertake data reduction. As such, this paper should be used in conjunction with the aforementioned guides as it assumes general knowledge of the TESS reduction process and its respective vernacular. For the same reason, AstroImageJ (AIJ) is the photometry software utilized in this paper. This paper will address the TESS steps chronologically. Image titles are censored as mandated by TESS publication policies. 


\section{BACKGROUND}

TESS is on the cutting edge of exoplanet research. The mission's goal is to deliver fifty Earth-like planets. With such a grand objective, the novelty of this exciting data benefits thousands of scientists all over the world. The possibilities of the data are endless. As such, research foundations such as the Boyce Research Initiatives and Research Foundation (BRIEF) are so vital to TESS as they provide the education necessary to train the next generation of scientists who will unpack the mysteries within the data.

BRIEF is a member of the SubGroup1 (SG1) for the TESS. In April 2018, when the NASA/MIT team launched TESS with its first year's survey being in the southern skies, BRIEF students were already preparing to perform exoplanet transit data reduction by the use of discovery surveys (KELT, K2). BRIEF is a global Sky Partner for LCO (Brown et al., 2013) that robotically operates $0.4 \mathrm{~m}$ telescopes sited at three observatory locations in the southern and northern hemisphere. These LCO systems with 6303 SBIG CCDs are well suited to making light curve measurements down to a light curve depth of about five parts per thousand reduction in the star's stellar flux. By the fall of 2018, BRIEF was making TESS observations for the students to perform the data reduction on to submit to the Exoplanet Follow-Up Observing Program (TESS ExoFOP).

The mission of the SG1 worldwide group of groundbased small telescope observers is to, as described by TFOP, "identify false positives due to nearby eclipsing binaries that contaminate the TESS image of a candidate transiting planets." These false positives are common because the TESS images are taken with a resolution of 21 arcseconds per pixel. Often there is light flux from several stars that could create the observed light curve falling on or near that large TESS pixel. Therefore SG1 observers use ground-based telescopes to take higher resolution images in order to discern which star(s) are causing the light curve and recommend their nature. Once a star and the light curve created by the exoplanet is vetted by SG1, the candidate is then observed by the subsequent subgroups using means that are ever more sophisticated. All five subgroups compose a winnowing process that aims to reach the TESS program's goal, fifty rocky planets for future study.

In order to streamline the data reduction process, BRIEF uses the Our Solar Siblings (OSS) Pipeline. The OSS Pipeline further calibrates the CCD images, removes artifacts such as cosmic rays, and plate solves each image.

In a similar vein, after the initial TESS observations in late 2018, Dr. Conti and the TESS program management developed two important enhancements needed for the TESS ExoFOP SG1 data submissions. The first is a macro incorporated into AIJ that facilitates the measurement of the potential false positive light sources to assess their influence on the light curve. The second is the TFOP SG1 Observation Guidelines, a thorough statement of the requirements for submitting data to the TESS Follow-up Observing Program (TFOP).

Lastly, using the tools and procedures outlined by Dr. Conti's AAVSO course as well as his Practical Guide to Exoplanet Observing as a foundation, BRIEF has developed a newer guide. It incorporates the more recent developments specific to TESS observations, notably the AIJ macro, the TFOP SG1 Observations Guidelines, and the OSS Pipeline. The current "AIJ Guide for LCO - TESS Observations" defines the procedures referenced in this paper and is available for download at BRIEF's website.

\section{PREPARATION PHASE}

AIJ frequently changes and updates its software, so when AIJ is first opened, the system should be updated to Daily Build. The current Daily Build version of AIJ allows users to use its automated NEB (Nearly Eclipsing Binary) function that will come in handy later on. It's a quick step that will save a lot of time.

While working through the analysis, the TESS Transit Finder (TTF) (Figure 1) comes up a lot. It is a web based tool and database for finding information on exoplanet candidates that TESS has discoveredcontaining important information that will be used throughout the analysis. The TTF will be the single most important resource while conducting this analysis, so it's important to understand the information it contains before the analysis begins. To help make the analysis run smoother, data is documented from the TTF onto a blank template to organize all the data. Figure 2 shows a sample TTF Organization Sheet.

When the images are imported, make sure "virtual stack" is selected. This ensures that AIJ operates on large data sets without running out of memory, speeding up the process of importing the images.

After the images are imported, an important first step is to go through the images to weed out "bad" images, such as images that have random streaks 


\begin{tabular}{|c|c|c|c|c|c|c|c|c|c|c|c|c|c|c|c|c|}
\hline $\begin{array}{c}\text { Local } \\
\text { evening } \\
\text { date }\end{array}$ & Name & TOI & $\underset{\mathbf{m a g}}{\mathrm{V}}$ & $\underset{\text { mag }}{\mathbf{T}}$ & $\begin{array}{l}\text { Start- } \\
\text { Mid } \\
\text {-End }\end{array}$ & Duration & $\begin{array}{l}\text { BJDTDB } \\
\text { start- } \\
\text { mid-end }\end{array}$ & $\begin{array}{l}\text { Elev. } \\
\text { at } \\
\text { start, } \\
\text { mid, } \\
\text { end }\end{array}$ & $\begin{array}{l}\text { Az. } \\
\text { at } \\
\text { start, } \\
\text { mid, } \\
\text { end }\end{array}$ & $\begin{array}{c}\mathrm{HA} \\
\text { at } \\
\text { start, } \\
\text { mid, } \\
\text { end }\end{array}$ & $\begin{array}{c}\text { RA \& } \\
\text { Dec } \\
(\mathrm{J} 2000)\end{array}$ & $\begin{array}{l}\text { Period } \\
\text { (days) }\end{array}$ & $\begin{array}{c}\text { Depth } \\
(\mathrm{ppt})\end{array}$ & Priority & $\begin{array}{c}\mathbf{R}_{\text {planet }} \\
\left(\mathbf{R}_{\oplus}\right)\end{array}$ & Comments \\
\hline \multicolumn{17}{|c|}{ Sun. 2019-02-10: Nautical twilight 2019-02-10 21:30-2019-02-11 06:24 local time / 2019-02-11 00:-30-2019-02-11 09:24 UTC } \\
\hline $\begin{array}{c}\text { Sun. } \\
2019-02-10 \\
\text { (local date) } \\
\text { Nautical } \\
\text { twilight } \\
00: 30- \\
09: 24 \\
\text { (UTC) }\end{array}$ & 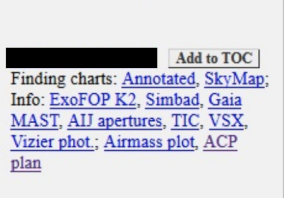 & & $\begin{array}{l}\text { Moon } \\
32 \% \\
@ 100^{\circ}\end{array}$ & 11.77 & $\begin{array}{l}03: 35- \\
04: 19 \\
-05: 03 \\
\pm 0: 16\end{array}$ & $1: 28$ & $\begin{array}{l}8525.650 \\
8555.680 \\
8525.711\end{array}$ & $\begin{array}{l}42^{\circ} \\
43^{\circ} \\
41^{\circ}\end{array}$ & $\begin{array}{l}12^{\circ} \\
358^{\circ} \\
344^{\circ}\end{array}$ & $\begin{aligned}-0.6 \\
+0.1 \\
+0.9\end{aligned}$ & $\begin{array}{l}08: 51: 32.35 \\
+17: 19: 11.4\end{array}$ & 1.11 & 7.9 & 4 & 8.8 & $\begin{array}{l}\text { event on } \\
\text { target and /or } \\
\text { check for } \\
\text { faint NEBs } \\
\text { within 2' and } \\
\text { bright NEBs } \\
\text { within full } \\
\text { FOV }\end{array}$ \\
\hline
\end{tabular}

Fig. 1. TTF information for a TESS object of interest.

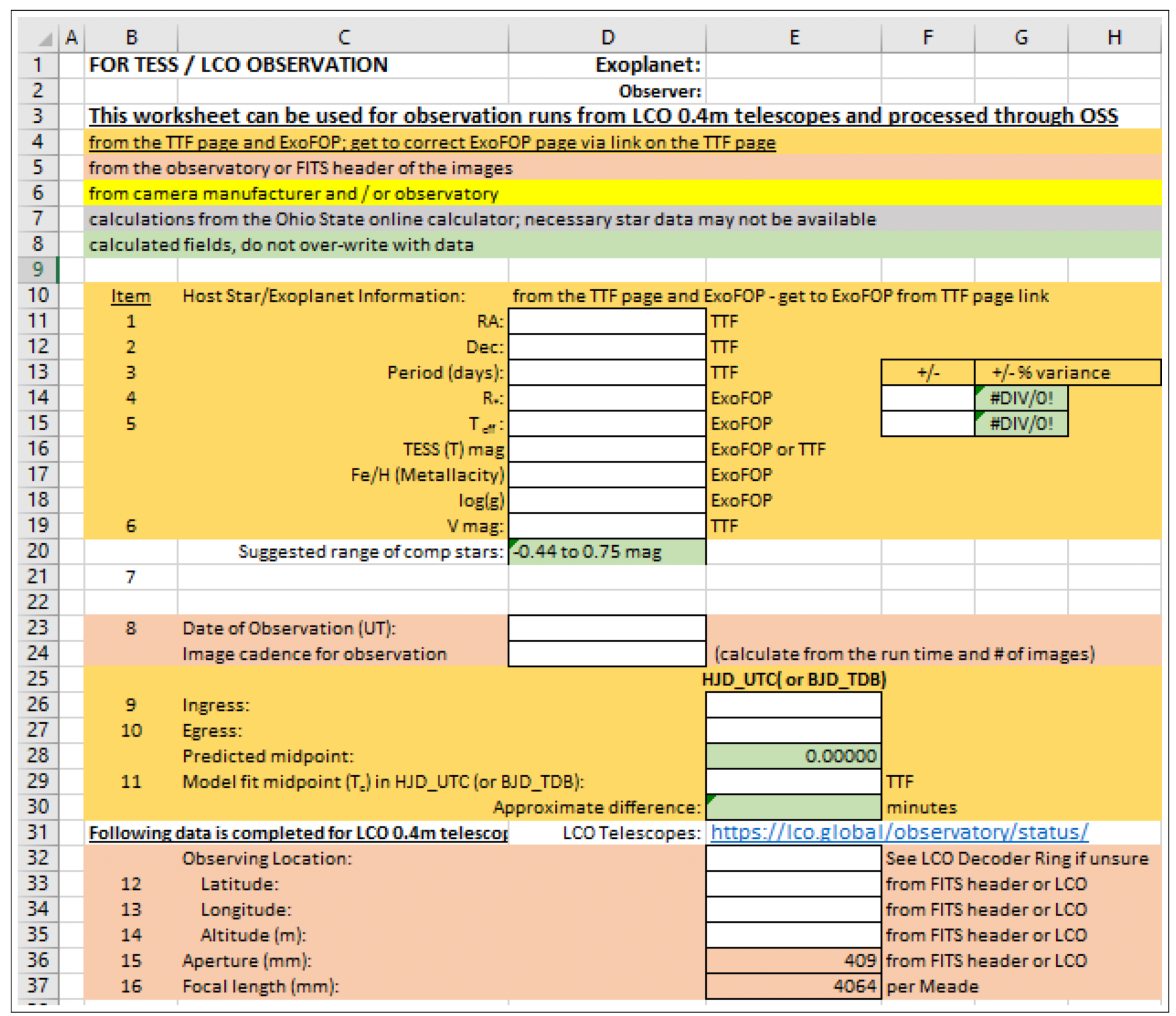

Fig. 2. TTF information sheet to organize the TTF data. 
or particles across them, or images that are blurry or misaligned with other images. An image with unwanted particles is shown in Figure 3). It also helps to use the two sliders (min and max) at the bottom of the screen to increase the brightness of the image via changing the contrast. To do this, locate the "peak" of the histogram on the bottom of the screen and place the two markers right around it (make sure under Scale, uncheck "use fixed min and max histogram values"). This will improve the readability of the image and help determine its quality. Figures 4 and 5 show a sample image before and after its saturation is improved.

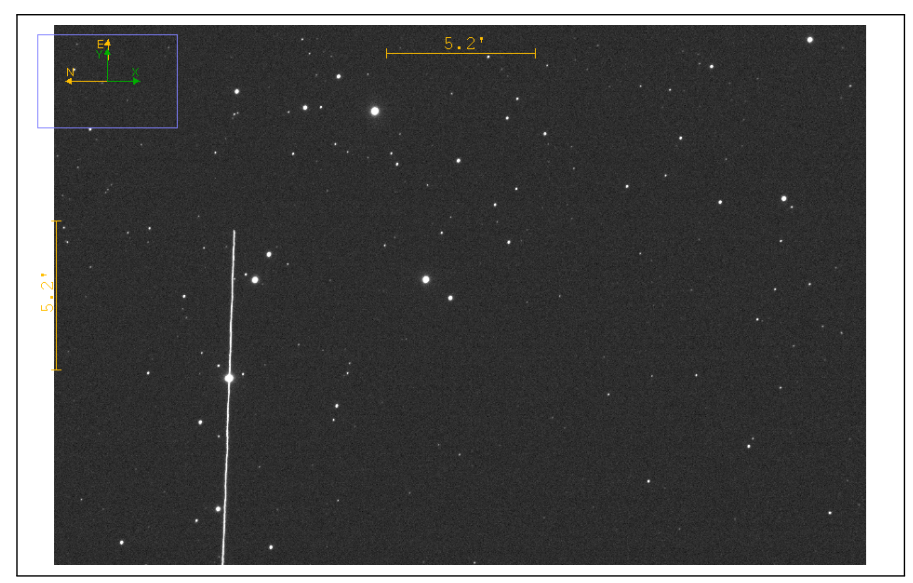

Fig. 3. Streaks like the one shown above are satellite trails.

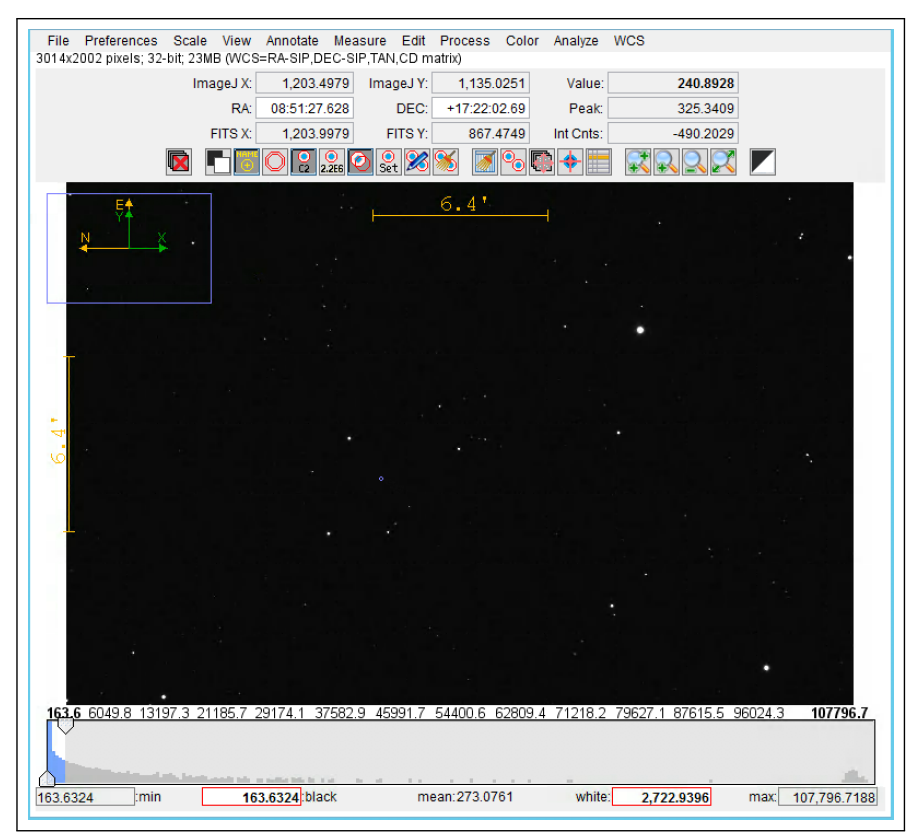

Fig. 4. Before utilizing the saturation scale: The image is very dark and essentially unreadable.

\section{CALIBRATION PHASE}

The images are calibrated in order to place the FITS header information onto the images so that the images' TTF data can to be inputted into AIJ in later parts of the analysis.

Firstly, the copies of the science images should be used (as opposed to the original files) because often, especially when first learning the process of TESS analysis, mistakes are made. For example, the calibration phase may be done incorrectly or the wrong images may be "quarantined."To mitigate these risks, it is prudent to use the copies of the science images.

After selecting the "DP" icon on the AIJ toolbar, locate and select the "wrench:" this is the General FITS Header. Next, select the "edit" box on the main image profile, and then locate the "edit FITS header" window as well. Note that the FITS header isn't actually being edited. Instead, the "edit FITS Header" table must be checked to see if it matches up with the General FITS Header that pops up when the "wrench" is selected. For example, if Target Name and Keyword, Target RA Keyword, Target DEC keyword, etc. match up for both headers, AIJ will correctly use these keywords to find information about the images when they calibrate (Figure 6). If the keywords don't match, AIJ won't be able to find the necessary information and will not be able to properly complete the calibration. If this is the case, then edit the information on the General FITS Header to match those on the "edit FITS Header."

The choice of "negate" for "Observatory Longitude" in the "FITS Header Input Settings" comes down to the location of the observatory where images were taken. If the observatory was west of the prime meridian and east of the international dateline (location can be found in FITS Header editor), then its longitude should be negative. Thus, if the FITS header displays longitude as a positive number, then the "negate" box should be clicked to indicate to AIJ that the number found in the FITS header should really have a negative sign associated with it. Observatory Latitude negate box should be unchecked.

These FITS headers contain "Right Ascension and Declination (RA and DEC)" keywords. RA and DEC make up the coordinate system of the sky. RA runs east-west while declination runs north-south. Their units are degrees, minutes, and seconds (the degree is too big of a unit to measure distances in the sky, so it needed to be split further).

For further clarification, "BJD TDB" stands for 


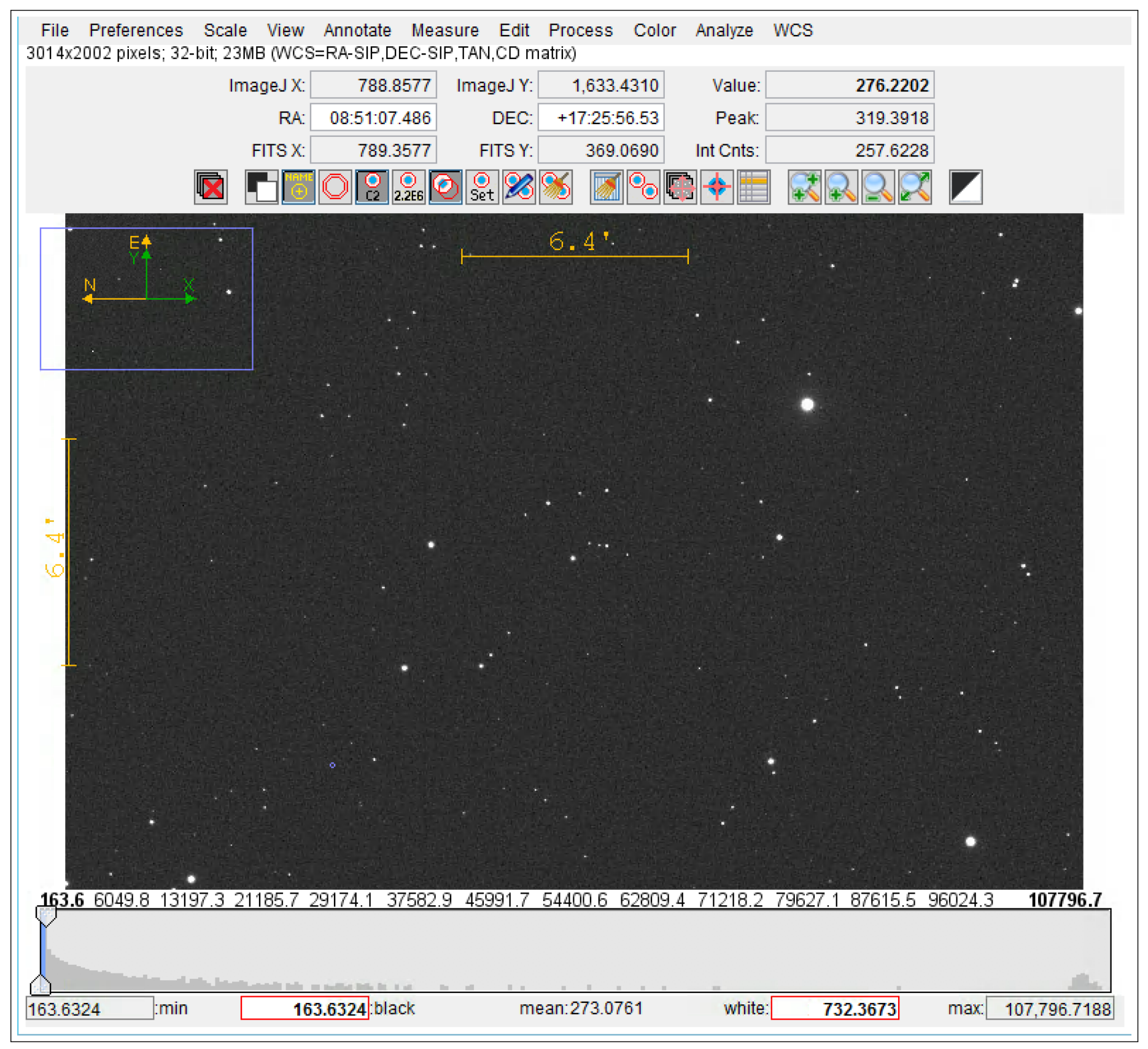

Fig. 5. After utilizing the saturation scale: The image is brighter and luminous sources are more visible. 


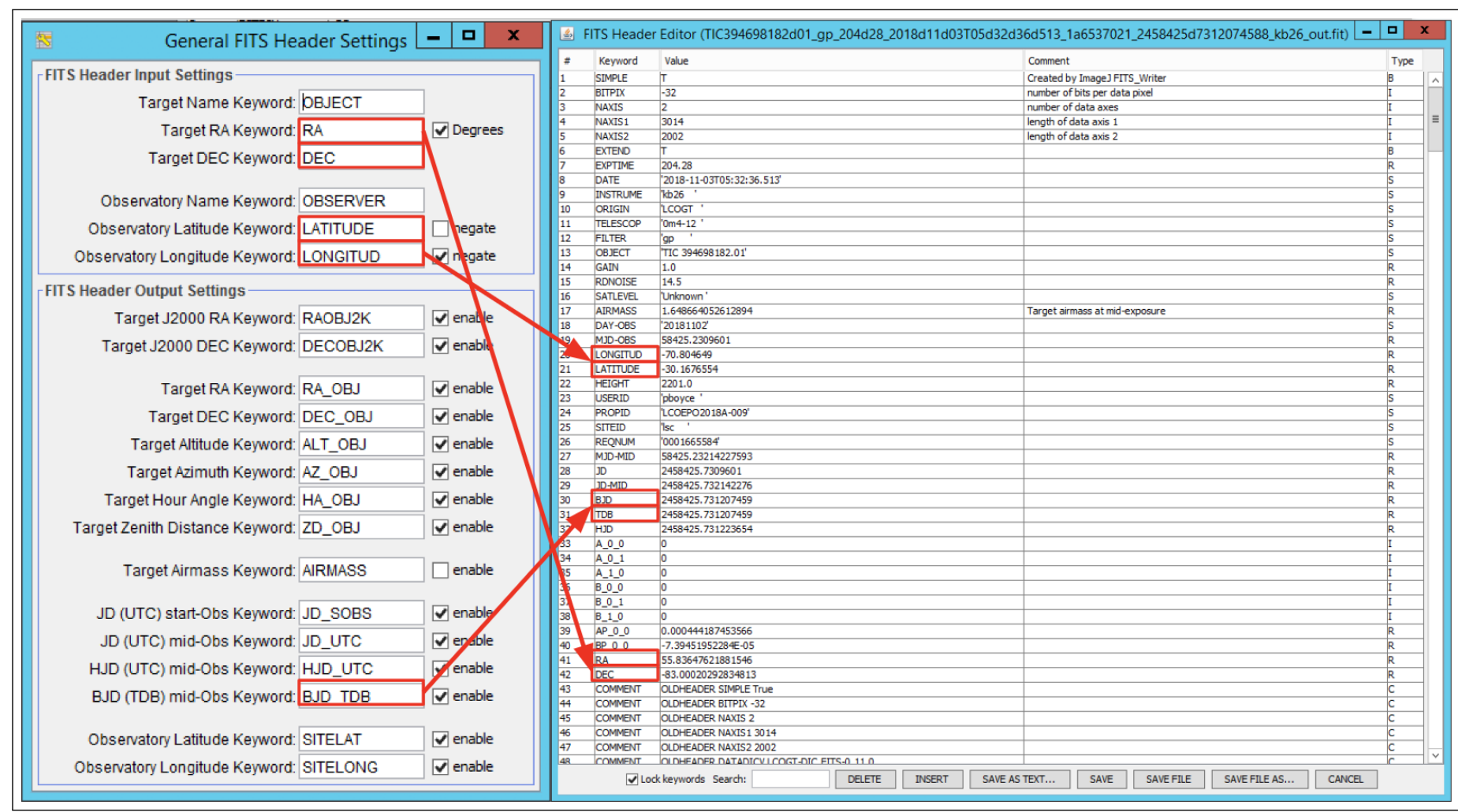

Fig. 6. Make sure that the keywords on both the "General" and "Edit" FITS Header Keywords match up.

"Barycentric Julian Date, Barycentric Dynamical Time." It's the standard time system for this exoplanet analysis, so make sure that this is enabled on the General FITS Header Settings.

Lastly, if the "Target Airmass Keyword" is disabled when the images already have data for Airmass, AIJ will override the data, creating all kinds of mistakes. For most users, there will almost always be Airmass data since most "fits" images have the Airmass header item. As such, "Target Airmass Keyword" (Figure 7) can be un-checked.

\section{Target Airmass Keyword: AIRMASS $\square$ enable}

Fig. 7. This setting is disabled.

Since the OSS Pipeline already plate-solved the images, "plate-solved" should be unchecked on the CCD Data Processor. Furthermore, on "Target Coordinator Source" and "Observatory Location Source," selecting "FITS Header target RA/DEC (J2000)" and "FITS header latitude and longitude." This basically tells AIJ that the necessary data is already on the FITS header. Usually this will be the case and other options won't need to be selected and this data won't need to be manually inputted. Also, make sure the polling interval is set to 0 since AIJ doesn't need to be working in "real-time." In "real-time," non-zero polling interval values are used and images are imported throughout the exoplanet analysis process.

Finally, after hitting START, the calibration of the images will begin, and a log should show the process. If successful, the log will say "finished" and the calibrated files will be in a folder that's inside the folder of the uncalibrated images.

Lastly, image orientation has some significance when submitting to Exo-FOP TESS: North should be up and East should be left. If this is not the case, open the first calibrated image, go to VIEW (on the top bar menu), and select Invert X or Invert Y, (or both, if necessary) until the correct orientation is obtained. Sometimes though, this problem of North and East alignment may be a problem with the telescope used for imaging. For example, North might be lined up in the negative $\mathrm{x}$ direction of the CCD detector, leaving no way to align North on top and East on the left or right. In this case, it's acceptable to put North to the left and East pointing down. This is a nuanced problem, so depending on who reviews the analysis, this may or may not be considered. 


\section{DIFFERENTIAL PHOTOMETRY PHASE}

One of the most important aspects of the Differential Photometry Phase is the ra.dec aperture file created from GAIA data. Steps on how to import this file can be found on Dennis Conti's TFOP SG1 Observation Guidelines. Essentially, this Gaia file finds all the stars in a 2.5 arcsecond circle of the target star, which allows the AIJ macro to check and see if these stars caused the TESS detection-most often these checks determine whether or not these stars are NEBs.

However, while the Gaia file checks for stars in this circle, this circle still needs to be manually created in order for it to show up on the image. First drag the ra.dec file onto the image. Then right click the target star. A box with settings will pop up. Figure 8 shows the correct input settings.

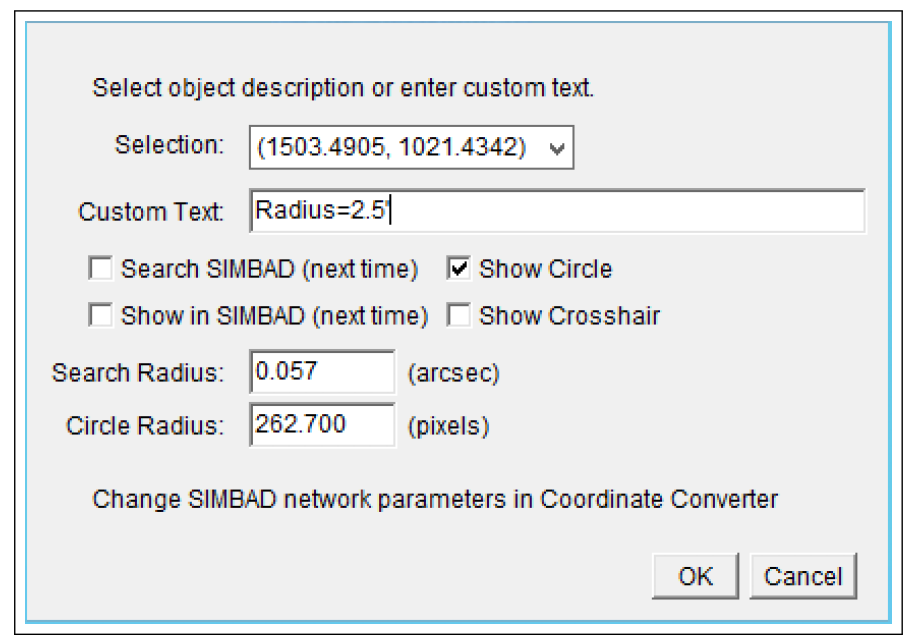

Fig. 8. These settings will create the correctly sized "annotation circle" to be used in the differential photometry phase.

The following steps describe how to select comparison stars.

1. Crosshairs are unselected because they might get in the way of the stars. Then click the "multiaperture photometry" button to open the aperture settings. If the "More Settings" button is selected, then the circle will disappear so the circle will need to be created again (basically, make sure all the Aperture Settings are established before creating the circle).

2. "Use previous X apertures" should be selected, but not "Use RA/DEC..." because AIJ automatically loads in the apertures created from the Gaia database.
3. Next, "Set Apertures" should be chosen. The field will show up again and select the target star. This will show the GAIA stars only, labeled with "T's."

4. Resume by selecting the comparison stars. Comparison ("comp") stars' peak counts (essentially how "bright" the star is) should be around that of the target star's. Comp stars should have about 8,000 peak counts and not more than 50,000 peaks counts. Figure 10 illustrates this notion of selecting comp stars based on their peak counts values.

At least six comp stars are necessary for analysis. Six is usually an adequate number as it leaves room for error in case a comp star's relative flux or other data comes out unusable for further analysis. Comp stars with similar peak values are the most desirable. However, if the number of viable comp stars "run out" before selecting six, which is usually the case, continue to select comp stars that are both above and below the target's peak value so that the data balances out (Figure 9). Lastly, while the preferred method is to use the comp stars' brightnesses to determine their selectability, be aware that there have been recent developments regarding alternative methods. Specifically, some cite using the stars' spectrums instead of their brightnesses.

Once the Differential Photometry phase has concluded, make sure a screenshot of the image with the 2.5 arcsecond circle, all the comp stars, as well as all the Gaia-generated stars is taken. The screenshot is one of the required TESS SG1 files for data submission. This can be done by either manually doing it using the computer's respective function, or by going to file, saving the image as a png, and selecting the "AIJ analysis" folder as its destination. Make sure that the file truly goes there, as AIJ can sometimes be clunky completing steps like these.

\section{TRANSIT MODELING PHASE}

First, the plot configuration (blank plot configuration template) is a life-saving file when it comes to the Transit Modeling Phase. It saves the settings of an entire plot-curves, labels, etc. As more analyses are completed, this file becoming increasingly important because it allows a researcher to easily go back and edit their plot and its settings without having to replot it from scratch. This can be extremely tiresome and inefficient. Thus, when starting new plots, use 


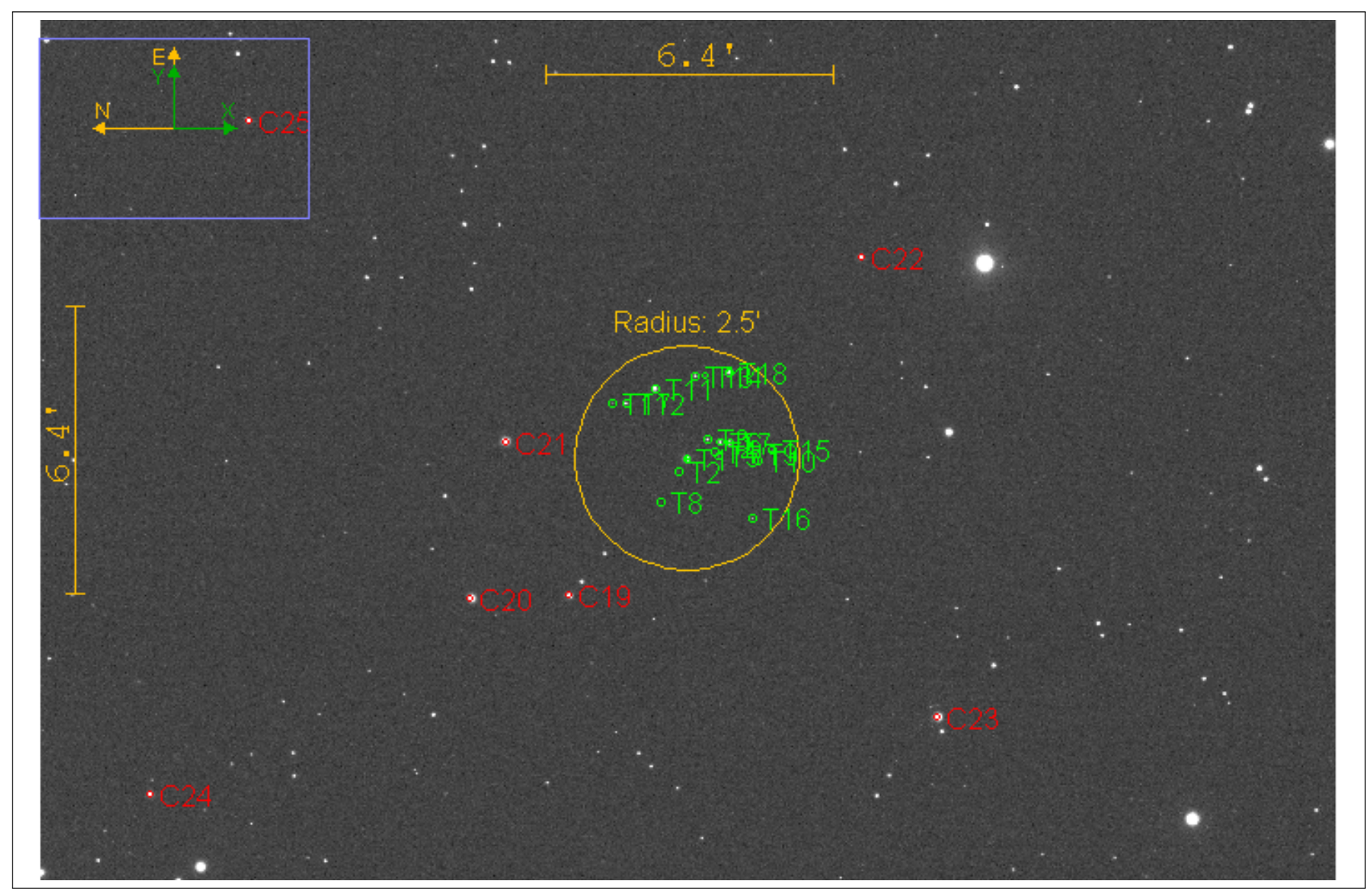

Fig. 9. This image shows the selection of reasonable comp stars as well as a 2.5 arcsecond circle around the target star, of which contains possible NEB's as documented by GAIA. 


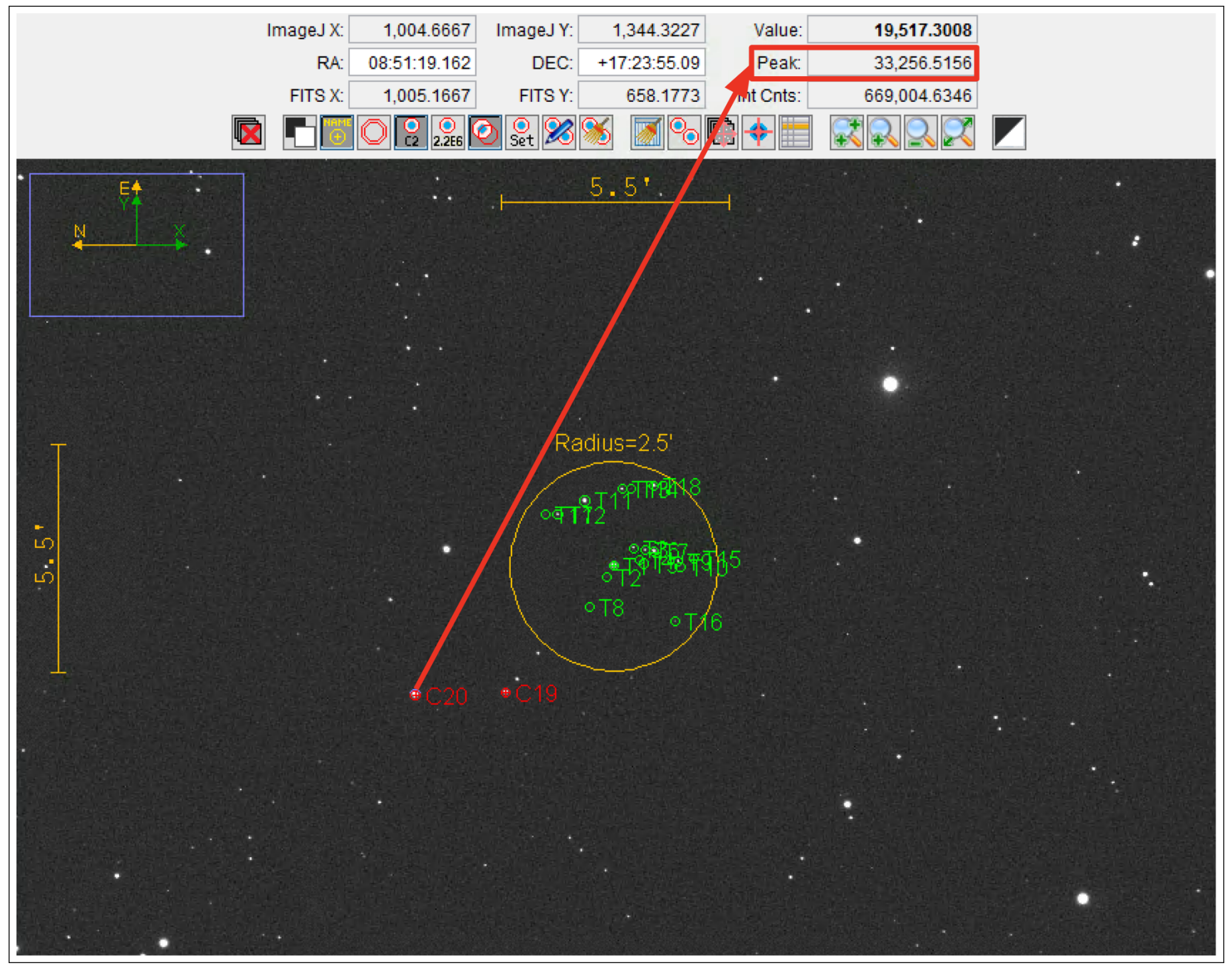

Fig. 10. In this case, the Target Star (T1) had a peak value of about 20,000 counts. Since no comp stars had 20,000 counts, comp stars should be chosen where the average of them is near 20,000. C20 and C19 were chosen as comp stars since peaks values were 30,000 counts and 11,000 counts, respectively. Accurate data analysis doesn't require peak values to be perfectly aligned since the data is rarely is. 
a blank plot configuration template so that data can be simply inputted without having to undo previous data. Also remember to save the plot configuration files during/after the process so that the plots can easily be updated even after closing AIJ.

When plotting curves, more often than not, there will be errors. For example, if the curve has outliers and certain data points need to be removed from the light curve, (though it should be kept as even poor data points are useful) there's a technique that can be used to fix it. First, note the BJD TDB (closest approximation) time for the outlier, then go to the sorted measurements table and look at the rows with that BJD TDB time. Next, select the row that has the T1 Rel Flux outlier data and delete it. Then rerun the Transit Modeling Phase. It should not take long since most of the last settings will have remained. Looking at Table 1, Slice (image) 63 would be removed.

\section{Table 1. Sample Data That Depicts an "Outlier" Plot Due to extraneous Rel. Flux.}

\begin{tabular}{cccc}
\hline Slice & BJD TDB & Airmass & rel flux T1 \\
\hline 19 & 2458525.604898 & 1.6973 & 0.114635 \\
41 & 2458525.605605 & 1.708598 & 0.114128 \\
57 & 2458525.607008 & 1.698978 & 0.114462 \\
63 & 2458525.607715 & 1.694244 & 0.74451 \\
88 & 2458525.60841 & 1.689581 & 0.114248 \\
99 & 2458525.609117 & 1.685014 & 0.115208 \\
\hline
\end{tabular}

Another method to consider when creating light curves has to do with the Multi-Plot Reference Star Settings (Figure 11). These settings deselect a comp star, thereby excluding it from the photometric analysis (model fitting). This can be particularly helpful if a comp star's data points are extremely erratic. Upon deselecting a comp star, the respective comp star become a "target" star, and the field of view will reflect this change. Furthermore, the light curves of all the other comp and target stars will become more or less fitted, as shown on the light curve. If light curves become less fitted, un-plotting a star via the Multi-Plot Y Data Screen may be a better option than using the Multi-Plot Reference Star Settings.

A small, yet interesting part of the transit modeling phase has to do with the ingress and egress times. Why are only the decimals inputted into AIJ and not the full ingress and egress BDJ TDB times? First, the ingress and egress represent the start and end times of a transit (when the exoplanet begins orbiting in front of the host star and after it orbits out of the host star's surface). The TTF only shows the last seven digits (including three decimals) of ingress and egress. The full time is listed on the FITS header. These times usually have the same integer values, which is why AIJ needs only their decimal values. However, sometimes the egress ends on the next day (one unit larger than the ingress). In this case, the egress values should be inputted as 1.XYZ as opposed to $0 . X Y Z$.

Furthermore, the ingress and egress are significant because sometimes the predicted ingress and egress will be different than the observed ingress and egress times. To determine the observed ingress and egress times, use the $\mathrm{x}$-values of the half-way points from the vertical predicted ingress line and the beginning of the flat horizontal line on the curve (do this for both predicted ingress and egress times). A difference between the predicted and observed ingress and egress usually means that the predicted time of transit is inaccurate; this should not have huge implications for determining false positives. Differences will affect the width of the light curve, however. (Figure 12).

In terms of general strategy to optimize efficiency during the Transit Modeling Phase, a useful technique is the "Half and Half Screen" (Figures 13 and 14) technique with the Plot of Measurements Screen and the Multi-Plot Y-Data Screen.

Basically, to see the Multi-Plot Y-Data screen's settings change in real time, split both screens in half so that they're next to each other. This allows for clear tracking of which settings correspond to which plots, reducing the chance of error.

Furthermore, for the Scale and "Then Shift" settings of the target stars on the Multi-Plot Y-Data screen, it is best to have them both be at Scale 1 . However, this may not be enough to show any real dip or curvature. Thus, a higher scale factor can be used as long as both rel flux T1 plots have the same Scale factor. The same applies to the comp stars, except that often times the comp stars won't have dips, so extremely high scale factors aren't as necessary.

Aesthetics have a significant place in this process. The plots that are created need to be read by future researchers, meaning that there's a standard of quality and readability that needs to be upheld. It's often best to keep light curves (and other lines) vertically dis- 


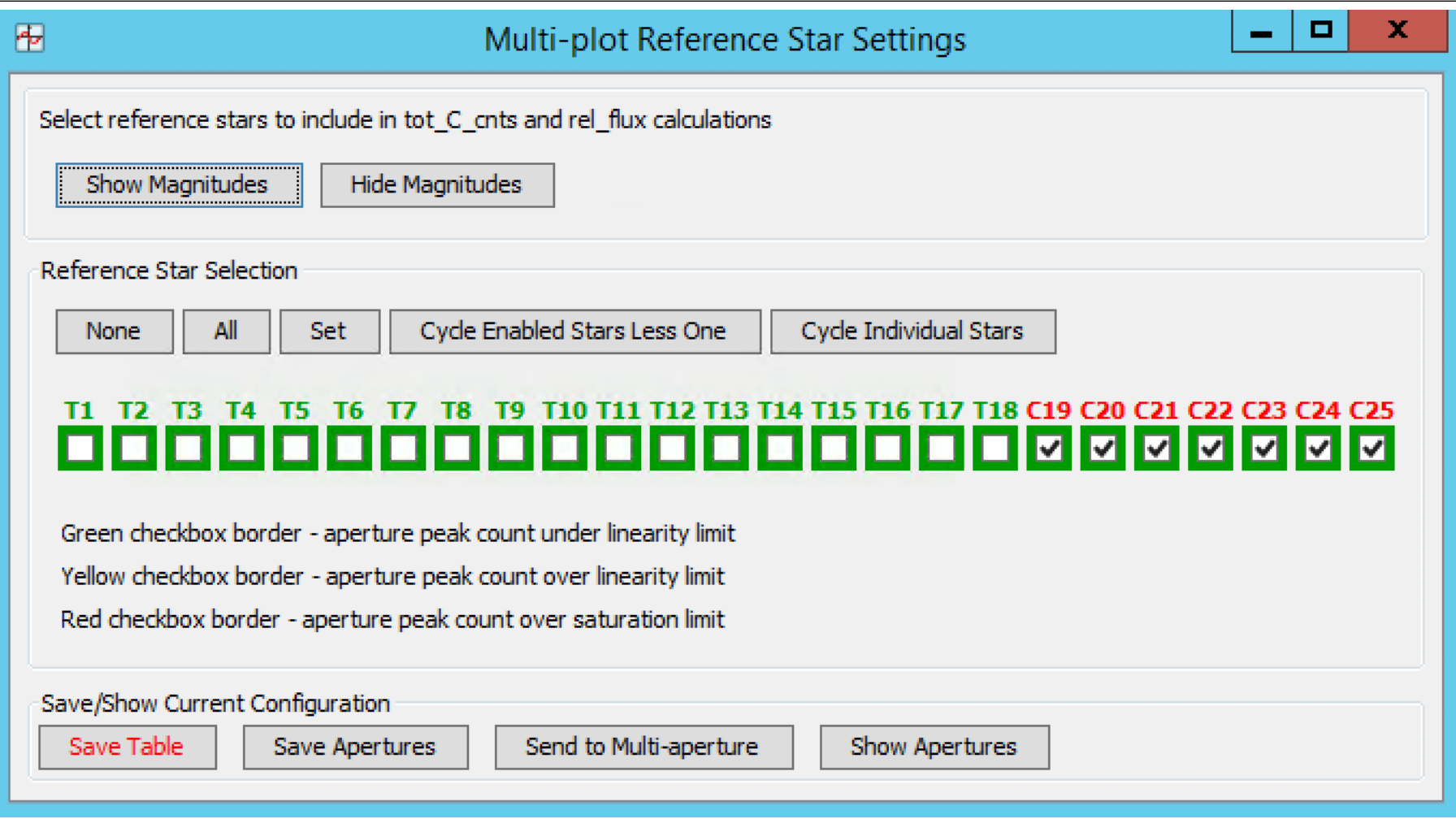

Fig. 11. Shown are 18 target stars and 7 comp stars. By deselecting one of the comp stars, it will subsequently become a target star, and will be exluded from the model fitting.

tanced from each other. The target star's light curves should be plotted on the horizontal lines marked by the y-axis in order to distinguish any dips that they might have. Also, a separate plot should be made for the comp stars as it'll expand the target star's plots, making the depth more visible. Figure 15 and its complementary comp star graph shown in Figure 16 show what a submitted light curve should look like in terms of readability.

Thus, one of the light curve plots should only have the target star's light curves and X(FITS) T1, Y(FITS) T1, tot C cnts, AIRMASS, Sky/Pixel T1 and Width T1 plots. The other plot should have just the comp stars (only about four or five are necessary for plotting). The comp stars that are selected for plotting should be the ones that don't change in brightness during the observation and that have the smallest variation. This can be seen by how well a comp star's plots match up with its "line of best fit" (Figure 18).

An important aspect of the Transit Modeling Phase is the Data Set 2 Fit Settings (Figure 17). Here, various information can be inputted to improve the accuracy of the light curve. For example, the period and estimated radius of the host star are information from the TTF that can be inputted here. The "Host Star
Parameters" are used to estimate the radius of the exoplanet. Limb darknening (LD) coefficients can also be inputted. LD coefficients are calculated using the $\mathrm{LD}$ calculator, where $\mathrm{Fe} / \mathrm{H}$ (Iron to Hydrogen ratio) and $\log (\mathrm{g})$ (the surface gravity of a celestial object measured in $\mathrm{cm} / \mathrm{sec}^{*} \mathrm{sec}$ expressed as a log of base 10) of a particular TESS object of interest (TOI) can be found on the ExoFOP - TESS Database. If these two pieces of data are not available, 0.3 is used for both LD coefficients.

There are a variety of beautiful colors on the MultiPlot Y-Data Screen. For target and comp stars, choose the colors freely (with reason: try to avoid making all the comp stars the same color). However, there are some specific color coordination procedures that must be followed. They only apply to non-target, non-comp star plots.

\section{CASE STUDIES/ANALYSIS OF LIGHT CURVES}

After the light curve has been created, the next step is to classify what kind of planet or host star has been analyzed. This part of the analysis is nuanced and is often regarded as the most difficult because it requires experience and well-justified subjectivity. In this next 
BRIEF (w filter, 52.34s exposure)

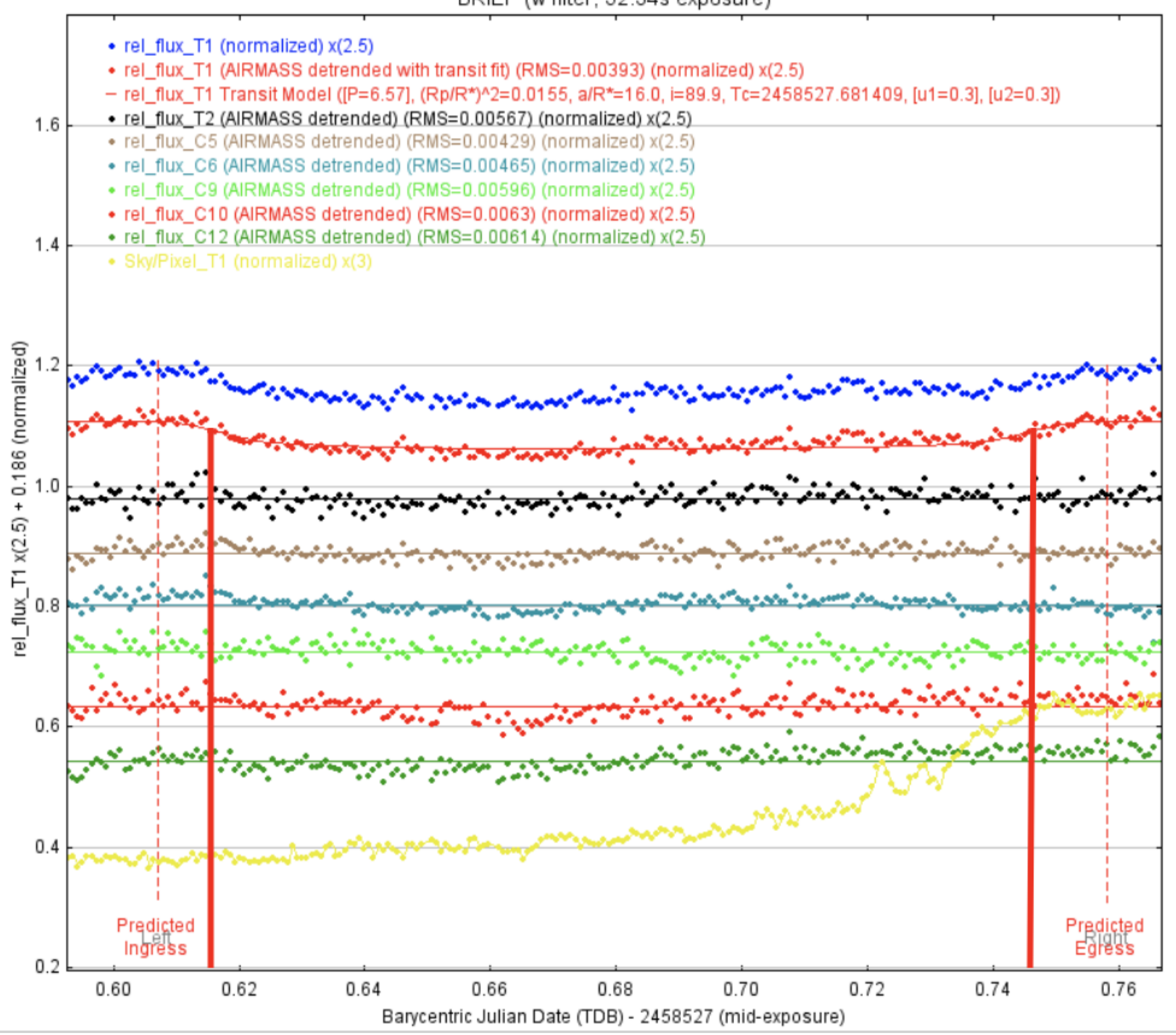

Fig. 12. The solid red lines represent the actual ingress and egresses. 


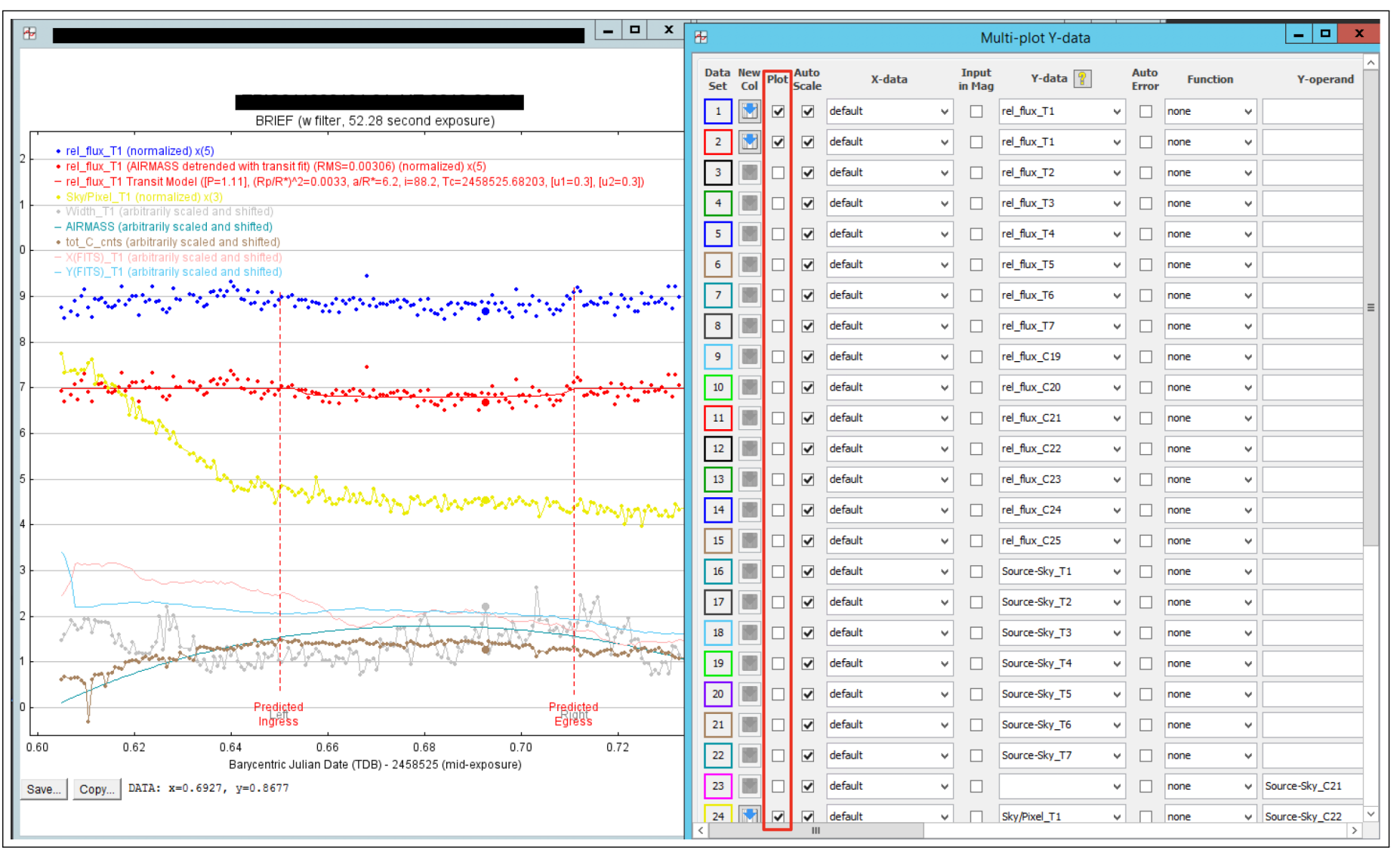

Fig. 13. A simple technique to use when modeling light curves. This will save time and increase accuracy.

section, a few common "case studies" will be covered along with how to handle them appropriately.

One possible cause of erroneous plots (plots that have seemingly no pattern in the data and lack form) is if the distance between the closest star and the target star (determined by going to the NEB folder created after doing NEB analysis, clicking on the first NEB depth plot, and then looking to see how far it is) is smaller than the aperture (aperture size can be checked from the seeing profile). Refer to Figures 19 and 20. This closest star could therefore contaminate the target star measurement, as its size isn't accurately accounted for by the given aperture. Thus, by using a smaller aperture, the amount of potential contamination of the target star measurement is reduced (using a smaller aperture size of half the original is usually safe). On the other hand, if the closest Gaia star is, for example, 20 arc-seconds away from the target and the aperture that is used is around 5 arcseconds, a second smaller aperture is not necessary. The used aperture size will need to be included the observation email though so that reviewers/future researchers are aware that the aperture was correctly accounted for.
One of the most important aspects in analyzing the light curve is the depth of the light curve. Normally, a shallow depth would indicate that "no clear detection" was found. But, if the predicted depth, as stated in the TTF (as shown back in Figure 1), is also a small number, then it cannot necessarily be concluded that "no clear detection" was found. Vice versa, if the predicted depth is significantly larger than the light curve's depth on the plot, then it may be appropriate to deem that "no clear detection" was found. "Detection" in this case refers to any detection of a possible exoplanet or eclipsing binary transiting across the host star.

NEBs are commonplace in this analysis. When one star orbits in front of its binary counterpart, a light curve is produced that is very similar to a light curve generated from planet orbiting across its host star. This is why NEBs are called "false positives." One way to determine whether data has a NEB is through the Dmag vs. RMS plot (Figure 21). RMS is essentially the amount light curve scatter, of which depends on the size of the photometric aperture. If there are any major outliers on the curve (which should have a general trend line of exponentiality), then these 


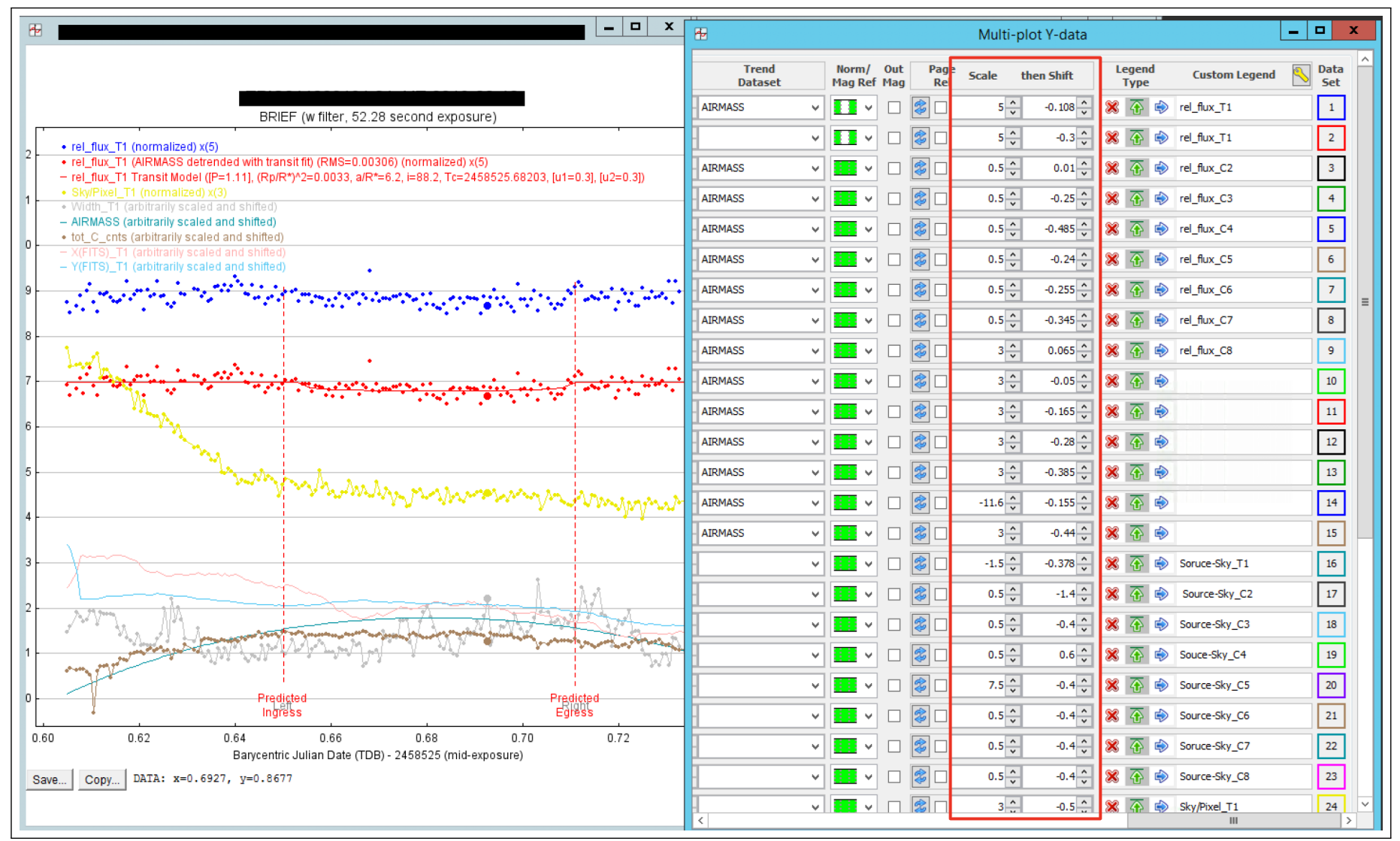

Fig. 14. This figure shows the right half of the Multi-Plot Y-Data Screen. The Scale and Then Shift settings for the curves are boxed in red. 


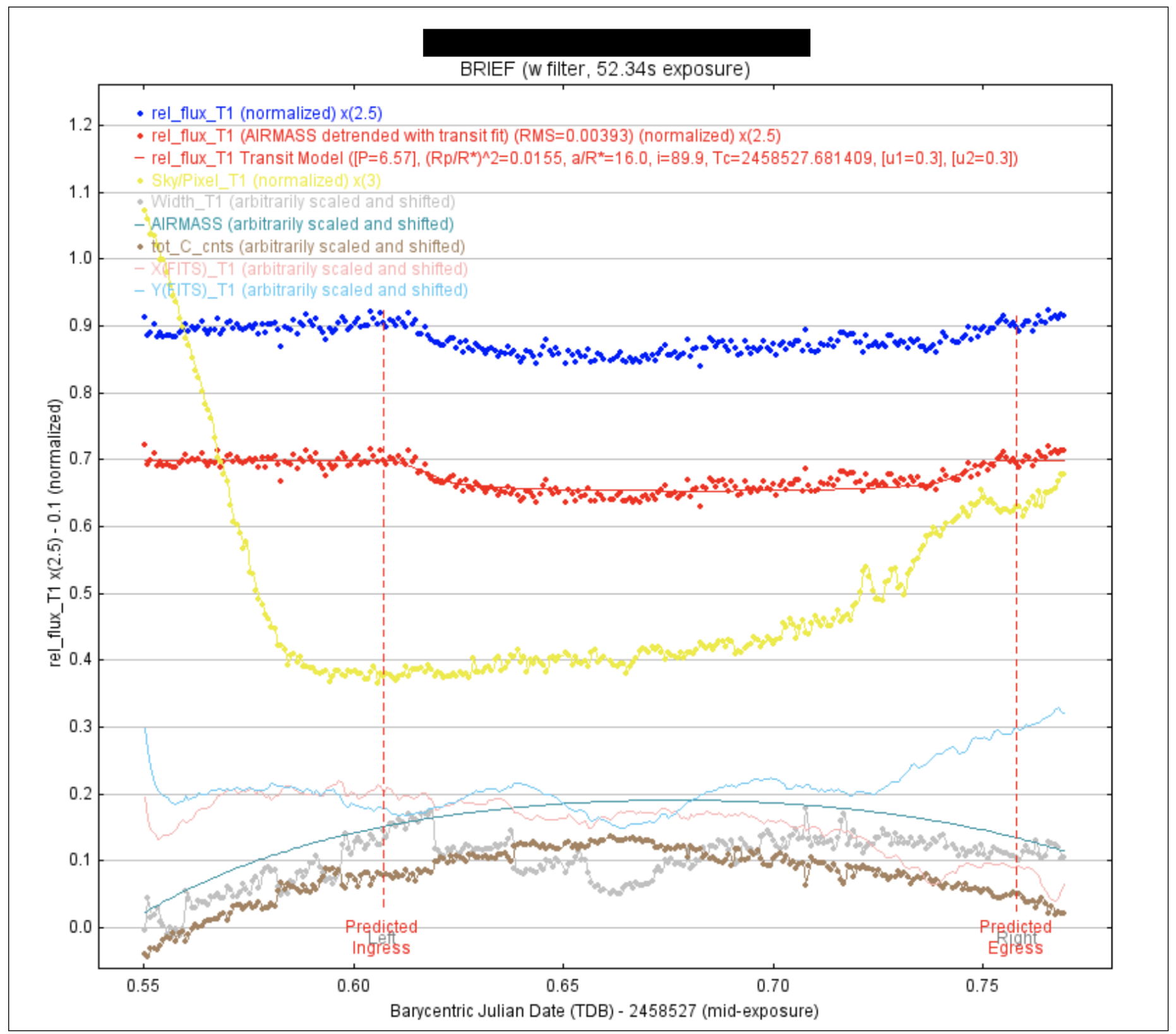

Fig. 15. Curves and other data should be spread out for readability purposes. 


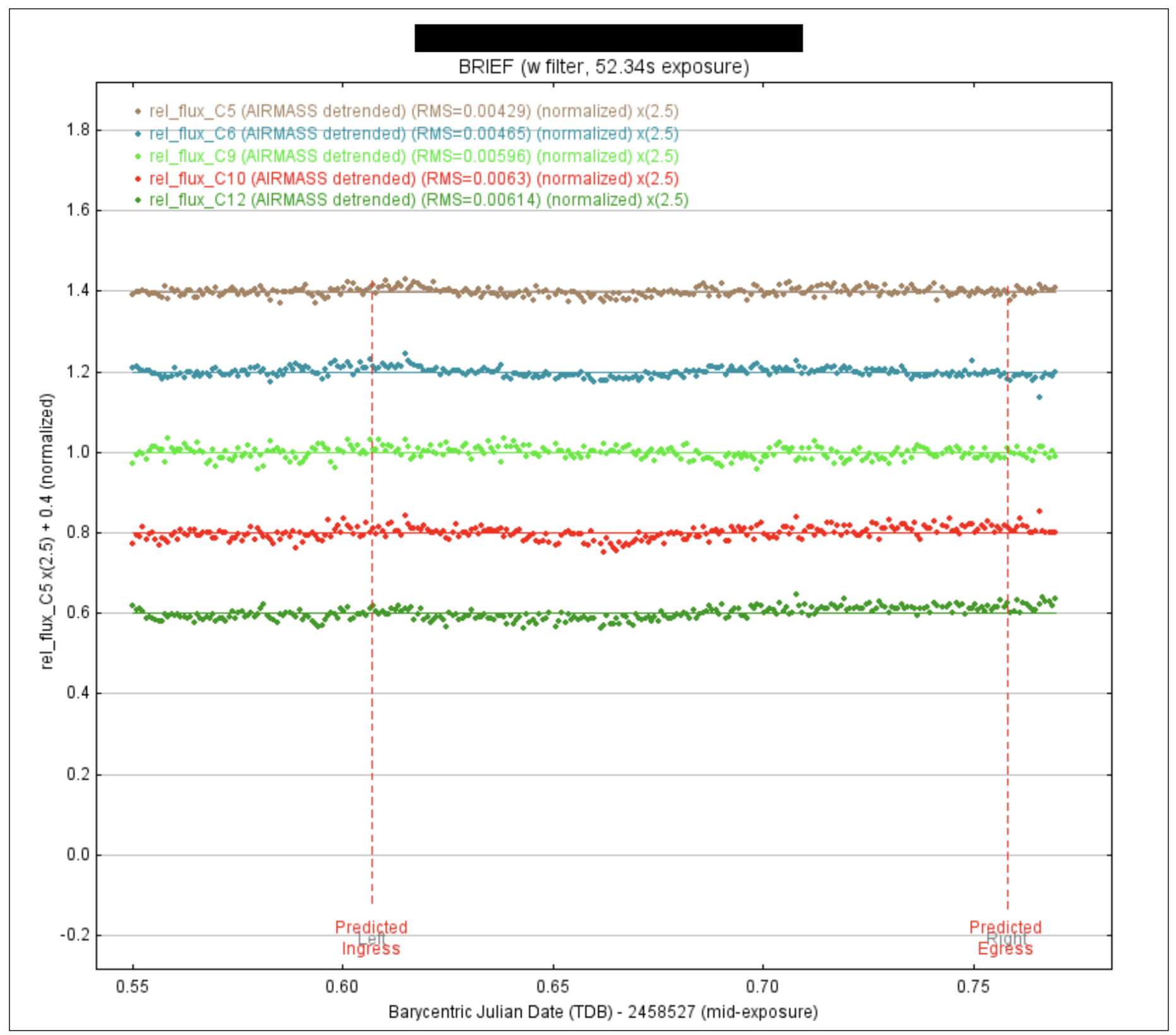

Fig. 16. The light curves of comp stars will often need to be graphed on a separate plot to avoid a single, cluttered graph. Mindful spacing applies. 


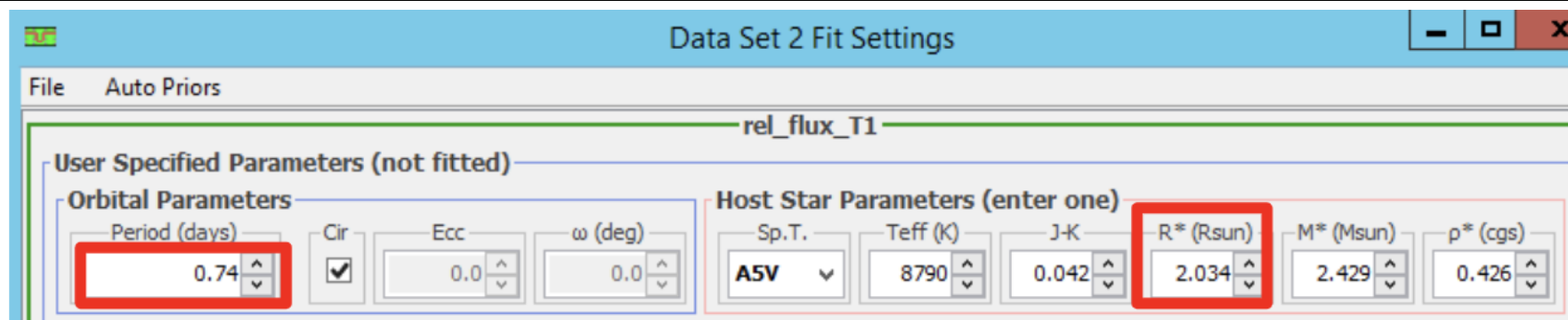

Transit Parameters

$\checkmark$ Enable Transit Fit

Parameter

Baseline Flux (Raw)

$\left(R_{p} / R_{s}\right)^{2}$

$a / R_{*}$

$\mathrm{T}_{\mathrm{C}}$

Inclination (deg)

Linear LD u1

Quad LD u2

Calculated from model

Detrend Parameters

\begin{tabular}{l|lr|l|} 
Use & \multicolumn{2}{c}{ Parameter } & \multicolumn{1}{c|}{ Best Fit } \\
$\square$ & AIRMASS & $\checkmark$ & -0.005793195535 \\
$\square$ & Width_T1 & $\checkmark$ & \\
\hline & Sky/Pixel_T1 & $\checkmark$ & \\
$\square$ & X(FITS)_T1 & $\checkmark$ & \\
$\square$ & Y(FITS)_T1 & $\checkmark$ & \\
\hline & tot_C_cnts & $\checkmark$ & \\
$\square$ & BJD_TDB & $\checkmark$ & \\
$\square$ & Meridian_Flip & $\checkmark$ & \\
\hline
\end{tabular}

Extract Prior Center Values From Light Curve, Orbit, and Fit Markers

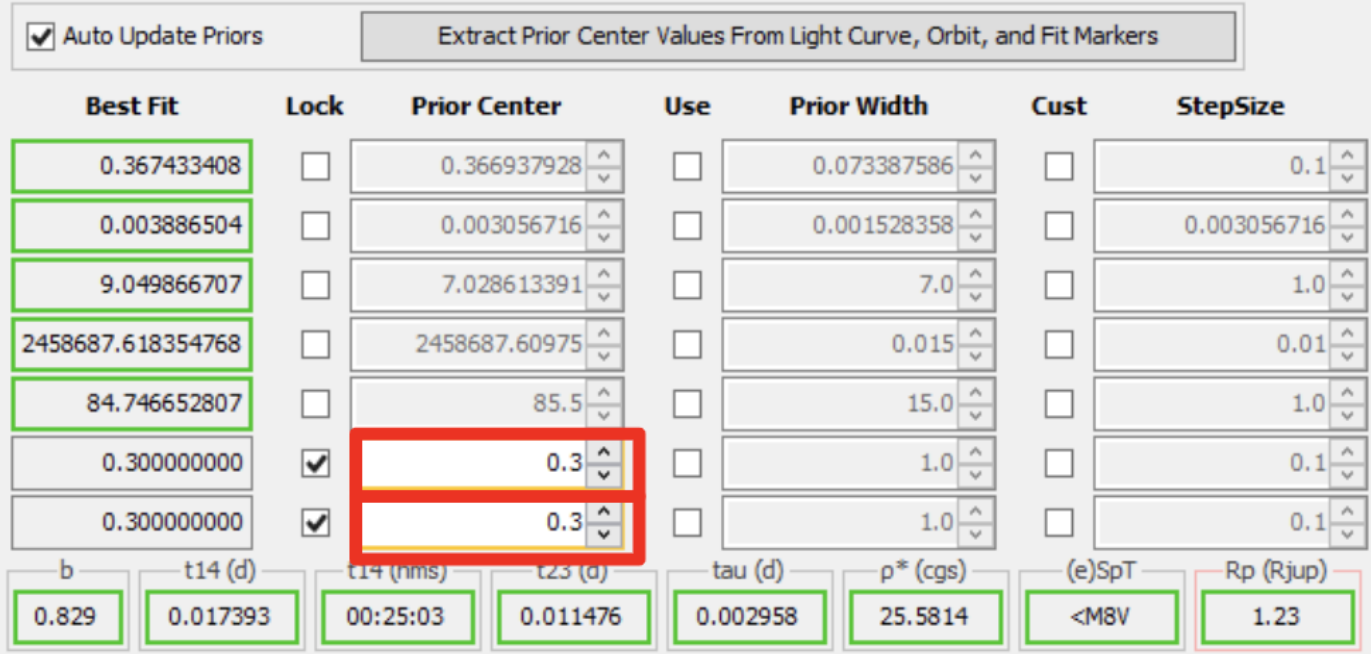

Fit Statistics

Fit Statistics

RMS (norm)

0.008084
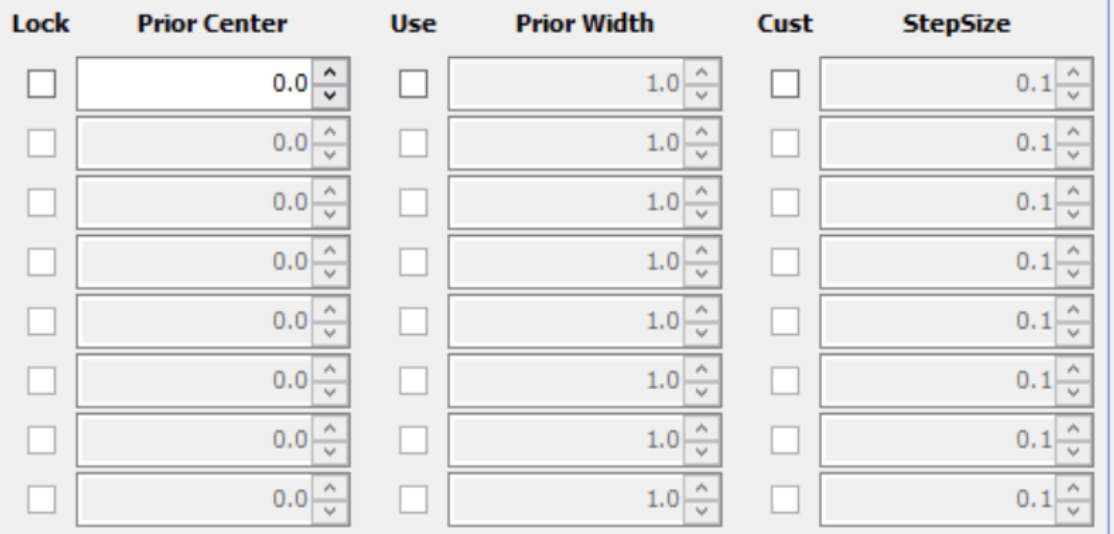

\section{Plot Settings}

$\checkmark$ Show Model

$\square$ Show Residuals

$\checkmark$ Show in legend

$\square$ Show Error
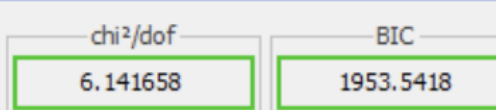

dof

chi $^{2}$

Fit Control-

Fit Control

Fit Update Options

Auto Update Fit Update Fit Now

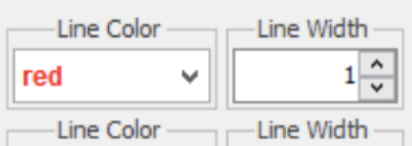

Line Color - Line Width -

magenta

$1 \hat{\imath}$

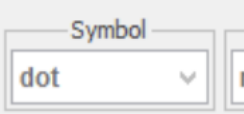

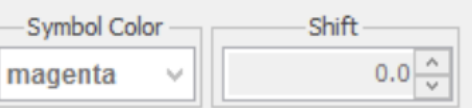




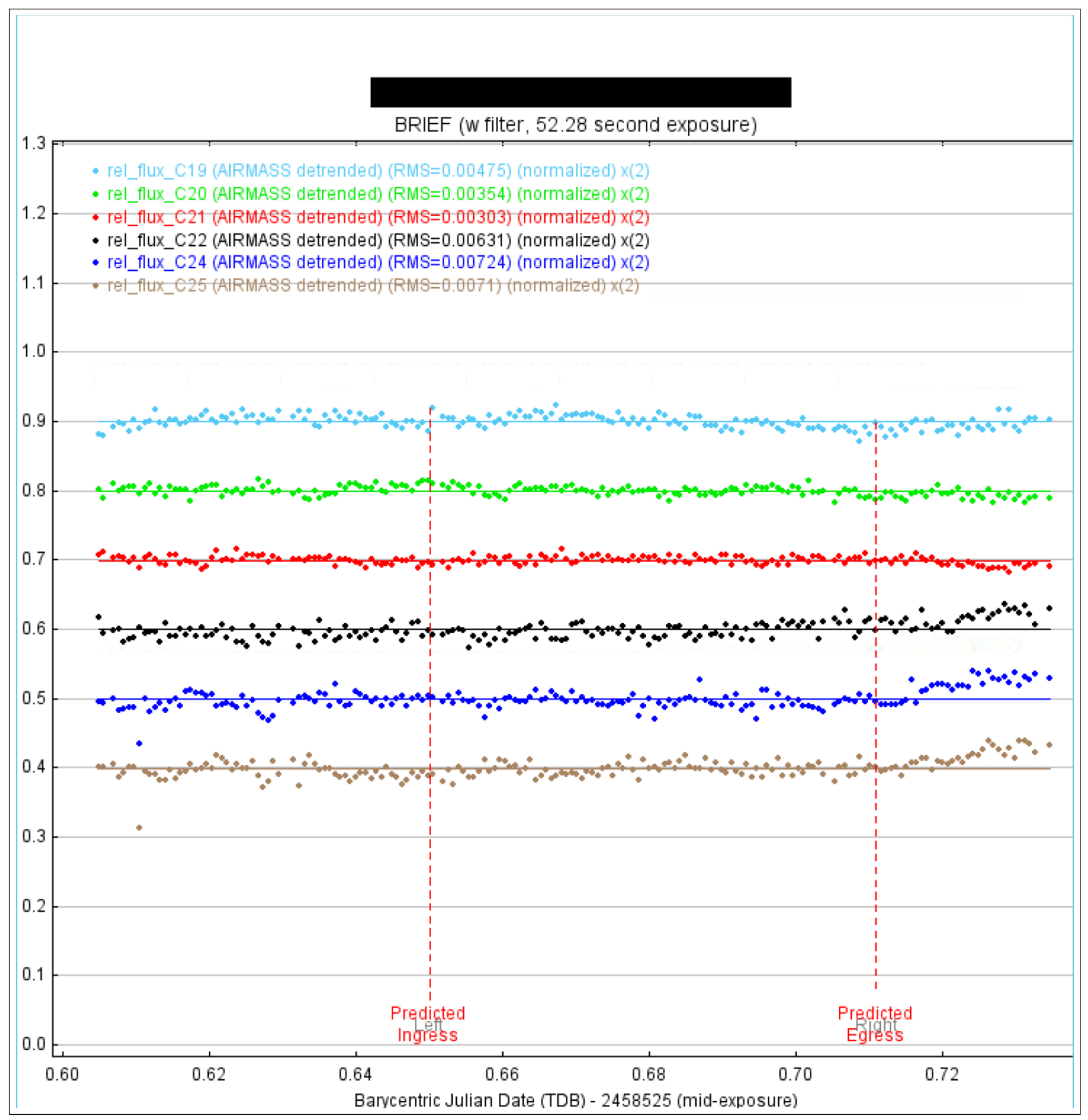

Fig. 18. Notice how the points nicely follow the line of best fit that runs through them. 


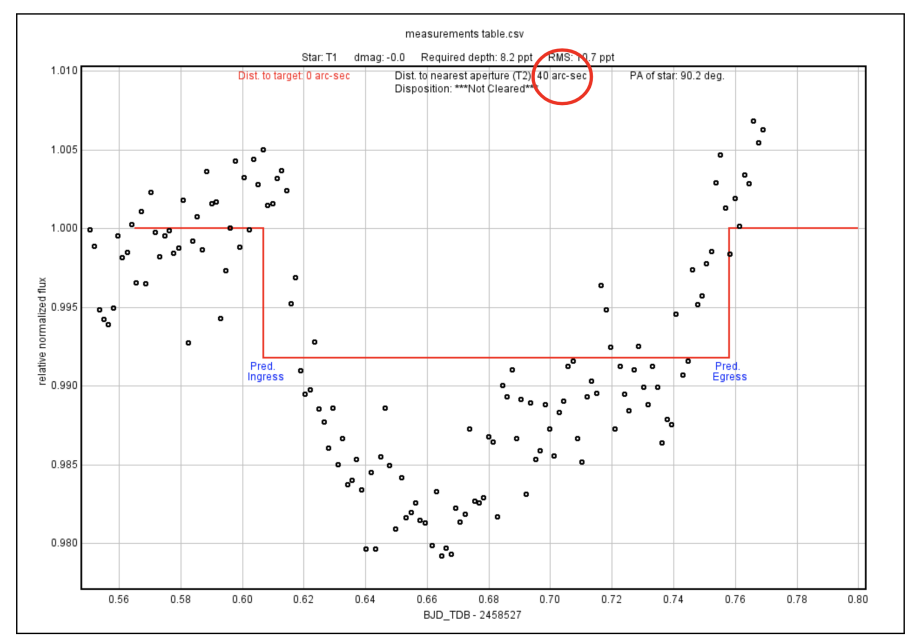

Fig. 19. The NEB Depth Plot of the Target Star. The distance, in arc-seconds, of the Target Star to the closet target star (from GAIA) is circled in red.

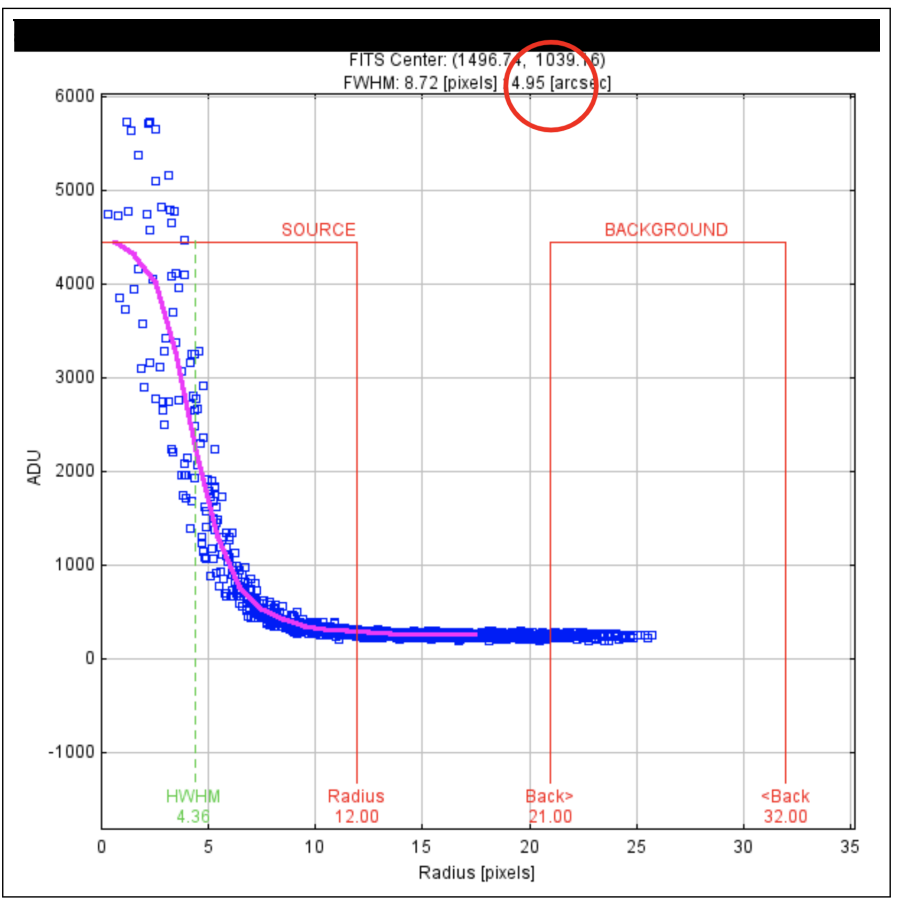

Fig. 20. The Seeing Profile shows the various radii of the aperture, inner, and outer annuli. The red circle shows the aperture of the instrumentation used to obtain the images. target/comparison stars are usually either NEBs, EBs, or other objects of interest.

Another way to discern NEBs is using the NEB Table (Figure 22). This list stars that are "Cleared, likely cleared,"too faint," or "not cleared." "Cleared" in this case means that it is cleared of not being a potential NEB, where "not cleared" means that a star could possibly be a NEB. Other keyword definitions are defined on the NEB Table.

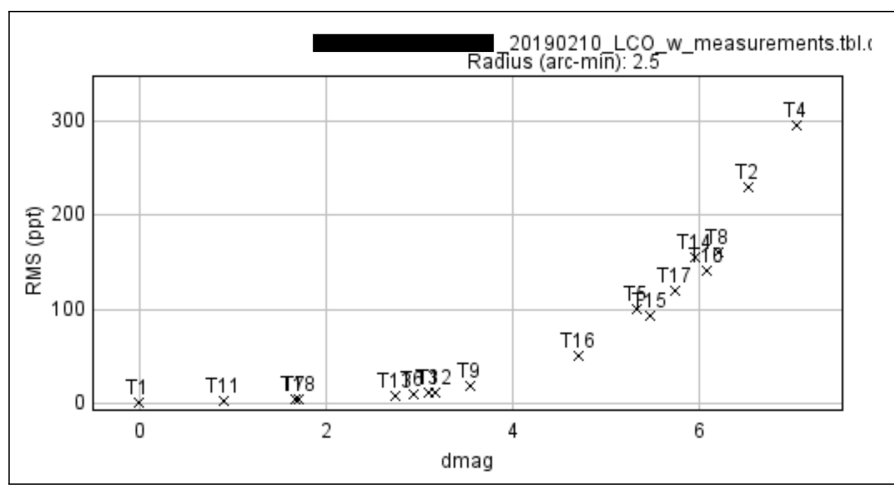

Fig. 21. Dmag vs. RMS Plot: Comp stars should compose an exponential curve; if certain stars deviate substantially, then they might be an NEB or EB.

\section{THE "REAL" CASE STUDIES}

In terms of categorizing a light curve and drawing conclusions, There are three predefined "cases."

The categorization of the target of interest is "Case 1 " if there's a clear "dip" on the target star's light curve and there aren't any false positives (as stated by the NEB Table or other aforementioned methods). It should look like the light curve presented in Figure 23. Case 1 often leads to planet candidates.

The target star should be categorized as Case 2 if non-target stars (outside the 2.5' radius) show an "event"-a dip in its light curve. Case 2 basically states that a NEB, or another possible planet candidate, is the cause of the event because it's outside the target star's region. Case 2 evidence, apart from just the light curve, can be found in the NEB Table, Dmag. vs. RMS Plot, and other resources mentioned in "Case Studies." However, be prepared for further investigation and validation from more experienced researchers.

If not Case 1 or Case 2, then the target of interest may be Case 3 which is defined as no events being detected by the target star nor with any checked, nearby 


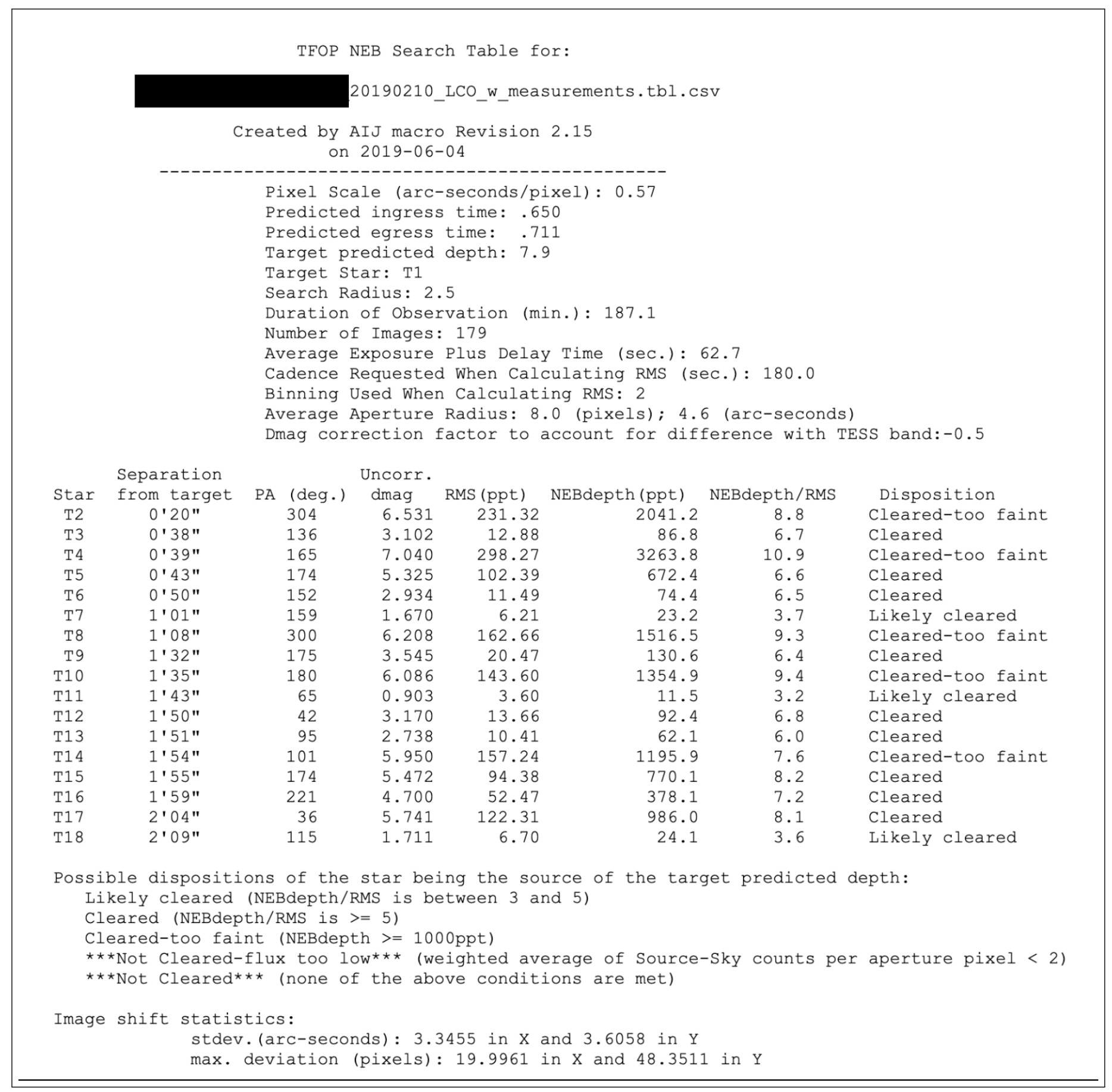

Fig. 22. NEB Table: Characterizes the likelihood of each of the GAIA-determined potential target stars being NEBs. 
stars. Case 3 is often deduced by process of elimination. Sometimes there may be an extremely slight dip in the light curve, causing the analysis to be in the grey-area between Case 1 and Case 2. In this context, it is recommended to state the findings instead of ascribing them to a certain case. Also, further judgements about classification of what analysis yields can be offered by review teams.

\section{NEEDED FILES}

After analysis is complete, a researcher has to compile twelve total files for submission to ExoFOP TESS.

- File 1: Measurements Table. Referenced in the Transit Modeling Phase. This table is created upon completion of the Calibration Phase.

- File 2: Plot Configuration File. This file is downloaded upon completing the Transit Modeling Phases (reference respective section).

- File 3: Apertures File. This file is created upon completing the Transit Modeling Phase and when "save all" is selected. Essentially, this file saves all the selected target and comp stars. Thus, if the Differential Photometry Phase needs to be re-done, the previous apertures will be available to be referenced.

- File 4: Light Curves. Reference Transit Modeling Phase. These curves are the heart and soul of the transit-photometry analysis.

- File 5: Field Image with Apertures. Refer to the Differential Photometry Phase. This is "screenshot" of the field with the GAIA stars, comp stars, and 2.5' arcsecond circle around the target star. This is necessary for TESS professionals to reference which comp stars correspond to various curves on the light curve plots.

- File 6: Plate Solved Image. This is not a screenshot, but rather the file itself. Since the OSS Pipeline will be utilized, the images will automatically be plate-solved.

- File 7: Seeing Profile. Refer to the Case Studies/Analysis of Light Curves Section. The seeing profile is created by right-clicking the target star (or by finding the respective option in the main toolbar of the image). The seeing profile provides aperture size recommendations.
- File 8: Notes and Results Text File. Refer to TFOP SG1 Observation Guidelines page 14. This file essentially includes the classifcation of the potential exoplanet along with any other conclusions made from the data.

- File 9: Delta Magnitude (Dmag) vs. RMS plot. This plot is a useful tool in determining potential NEBs. Reference the Case Studies/Analysis of Light Curves Section for a full description.

- File 10: NEB Table. This table is also instrumental in determining false positive. Refer to the Case Studies/Analysis of Light Curves Section.

- File 11: A zip file that contains NEB Depth Plots for comp and target (GAIA) stars. These plots show the "shallowest event required for that star to mimic the TESS predicted transit depth" (Conti, 2019).

- File 12: A Zoomed-in Field-of-View Image. This screenshot should show only the stars within the 2.5 arcsecond circle. The purpose lies in showing more detail to the individual brightnesses of each potential NEB within the GAIA field-ofview. Further explanations can be found TFOP SG1 Observation Guidelines.

\section{CONCLUSION}

This paper is a guide that helps SG1 TESS researchers avoid the common pitfalls of aperture photometry in AIJ. These pitfalls were documented by the BRIEF team who noticed the common mistakes that students were making in their exoplanet analyses.

Categorized into four different sections, this paper mirrors the set-up of Dennis Conti's A Practical Guide to Exoplanet Observing (Conti, 2018) in order to simplify the exoplanet analysis as well as to coordinate cross usage between this paper and other AIJ Guides.

\section{ACKNOWLEDGMENTS}

Special thanks to the Boyce Research Initiatives and Research Foundation for the opportunity to learn about this amazing field of exoplanets and introducing me to the cutting-edge TESS research. Further thanks goes to Dr. Dennis Conti in being a pioneer in making high-level exoplanet research more understandable and accessible to the masses. Also, big thanks goes to Dr. Scott Dixon for helping me on a day-to-day basis learn the specific methodology of 


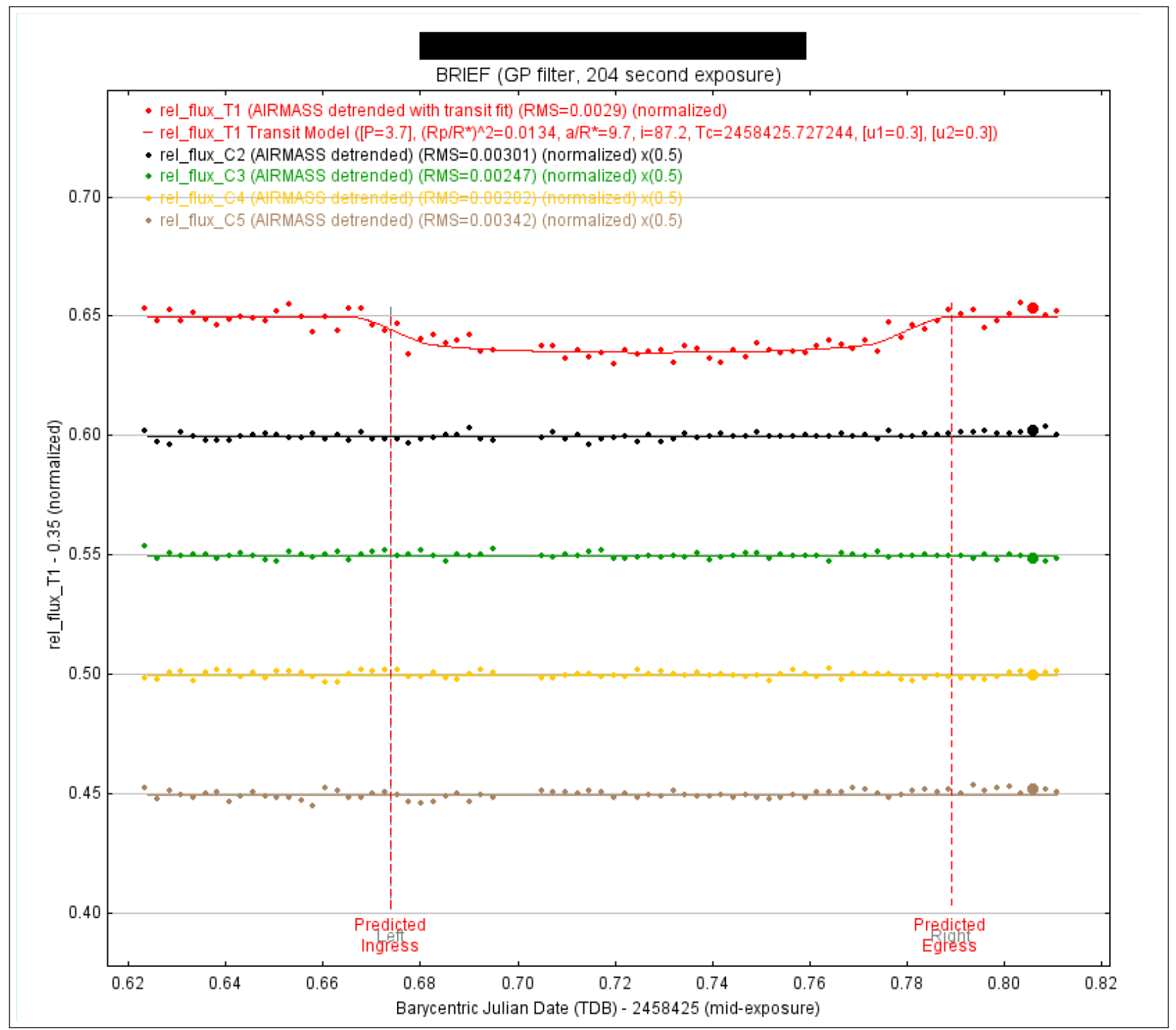

Fig. 23. Taken from real TESS data, this light curve represents an ideal situation where there's a nice dip and non-skewed comp stars. 
photometric analysis. Lastly, another big appreciation for Michael Fitzgerald for plate-solving the images through the OSS Pipeline, serving to streamline the research.

\section{REFERENCES}

Boyce, P., Boyce, G., Dixon, S., Conti, D., Falatoun, A., Wiley, E., ... Ha, J. (2019). The astroimagej guide for lco - tess observations processed through the oss pipeline.

Brown, T., Baliber, N., Bianco, F., Bowman, M., Burleson, B., Conway, P., ... others (2013). Las cumbres observatory global telescope network. Publications of the Astronomical Society of the Pacific, 125(931), 1031.

Conti, D. M. (2018). A practical guide to exoplanet observing.

Conti, D. M. (2019). Tfop sg1 observation guidelines.

Fitzgerald, M. (2018). The our solar siblings pipeline. RTSRE Proceedings, 1(1), 347-358. 